\title{
Virchows Archiv—an update, and plans for the future
}

\author{
Daniela Massi $^{1}$
}

Published online: 24 December 2016

(C) Springer-Verlag Berlin Heidelberg 2016

Dear readers,

I am most thankful to the European Society of Pathology (ESP) for the great honor of being appointed the new Editor-in-Chief of Virchows Archiv. As Associate Editor, a position and privilege that I held with great enthusiasm, dedication and loyalty, I experienced first-hand the tremendous effort and energy provided by Fred Bosman in guiding our illustrious and influential journal through a process of continuous and rigorous progress, in terms of scientific content and overall reputation. Fred Bosman, in a temporary role as the Senior Editorial Consultant, will continue to support the Editorial Team and to help increase the high standards of the Virchows Archiv.

Over the coming years, my chief priority will be to keep the momentum on the ongoing projects of Virchows Archiv and to increase the impact of the journal within our scientific community of European and worldwide pathologists. My editorial policy will be oriented towards attracting high-quality studies and cutting-edge clinical and translational research, offering a forum to the health community for research on diagnosing disease, including prognostic and predictive biomarkers for cancer. Interdisciplinary and collaborative investigations between pathologists, immunologists, geneticists, clinical oncologists and others will be particularly welcomed. Virchows Archiv must also warrant immediate and sensible attention to the rapid changes our discipline is facing and will continue to face in the next few years.

This ambitious task will be accomplished by the dedicated commitment and experience of a cohesive and motivated

Daniela Massi

daniela.massi@unifi.it

1 Division of Pathological Anatomy, Department of Surgery and Translational Medicine, University of Florence, Largo Brambilla 3, 50134 Florence, Italy

Editorial Team. In agreement with the Associate Editors, we will define strategic priorities and redefine the responsibilities of all team members. Cord Langner will take the position of Reviews Editor and will become the Deputy Editor-in-Chief from 2018. Hans Kreipe's mandate as Associate Editor ends alongside 2016, and George Netto will join as of 2017. Volkan Adsay, Sigurd Lax, Ales Ryska and Leticia Quantanilla complete the Editorial Team and will take responsibility for assigned manuscripts according to their field of expertise, providing the utmost scientific and clinical scrutiny. The main priority of Virchows Archiv, through the support of the Editorial Board and Reviewers, remains its commitment to publishing novel research that has been subject to a transparent and critical peer review process.

A journal's success depends in part on the quality and commitment of the contributing authors, and I underline my personal expectation of increased input from the ESP Working Groups. A closer stable collaboration between Virchows Archiv and the ESP Working Groups will be of primary importance, to ensure that the journal publishes high-quality, highly-cited multicentric studies and position papers in diagnostic practice, as well as in applied and translational research. The outstanding reputation of Virchows Archiv in human pathology will be maintained and part of what this requires is to increase its impact. To attain this goal, Virchows Archiv will publish invited contributions written by the Editors, members of the Editorial Board or by other experts in the field, including those belonging to ESP Working Groups, who will put the results of published articles in perspective, and outline future directions.

Recent developments implemented by Springer on their web platforms will certainly help to increase worldwide access to and visibility and distribution of our journal. Key data, including journal-level metrics such as citations and downloads, are now prominently placed on the article pages of SpringerLink. Changes made to the SpringerLink layout during 2016 include easy-to-use and manage PDF downloads, citation exports and 
social-sharing buttons, as well as the introduction of a fixed navigational bar that increases usability and the discoverability of desired information. In addition, SharedIt! provides authors with shareable links to view-only versions of their papers, links that can be posted anywhere, including social networks, institutional repositories, the author's own website and scholarly collaborative networks.

2016 saw continued collaboration between the publisher and Virchows Archiv. The journal's electronic table of contents (TOC) alerts are now subscribed to by more than 4000 pathologists worldwide. The 10 most downloaded articles from 2015 were made free-to-access and promoted within the scientific community. Virchows Archiv's Virtual Issue for 2016 was entitled 'Top Ten in Breast Pathology' and was circulated to increase usage of specific articles, raise awareness of the journal and gain new readers who might be potential new authors from 'emerging' markets. The Virtual Issue was circulated by Springer (to TOC Alert subscribers, as well as on Twitter) and by the ESP (to their member mailing list). The initiative was very successful and will be repeated in 2017. As a result of such initiatives and the continued contributions of the Editorial Board and readership, Virchows Archiv has successfully expanded its presence throughout the world. The global reputation of the journal is reflected in the breakdown of accepted manuscript by origin, manuscripts coming from Europe (64\%), Asia-Pacific (24\%), North America (10\%), Latin America (1\%) and the Middle East (1\%).
I wish to underline the journal's commitment to providing a fast and constructive peer review process. Throughout 2016, Virchows Archiv's average turnaround time from submission to the first decision was 23 days, and the average time from submission to the final decision was 58 days. The introduction of Springer's e-Proofing system has resulted in reducing the average turnaround time from editor acceptance to Online First publication to just 19 days. Two to three months after online publication the paper also appears in print. Virchows Archiv continues to be selective, publishing $30 \%$ of submitted papers. We also help $8 \%$ of authors to find a suitable journal for their paper when outside of Virchows Archiv's aims and scope by recommending the use of Springer's Transfer Desk.

I thank all 213 readers who provided feedback during our 2016 Reader Survey on the journal's standing and the changes implemented by the Editorial Team between 2012 and 2016. Your valued feedback is a key element in defining the journal's editorial strategy for the coming years.

I sincerely hope that all pathologists will continue to support Virchows Archiv in submitting the results of their research or readers of our cutting-edge papers. It is our aim to provide through Virchows Archiv insightful and stimulating information that will shape the future of our discipline and lead the way to extraordinary discoveries. 